\title{
Metabólitos sanguíneos de vacas de corte suplementadas ou não com sais de cálcio de ácidos graxos durante o período pré e/ou pós-parto
}

\author{
[Blood metabolites from beef cows supplemented or not with calcium salts of fatty acids \\ during pre and/or postpartum period] \\ M.F. Silveira ${ }^{1}$, J. Restle ${ }^{2}$, L.F.G. Menezes ${ }^{1}$, I.L. Brondani ${ }^{2}$, J.L. Nörnberg ${ }^{2}$, A.M. Callegaro ${ }^{2}$ \\ ${ }^{1}$ Universidade Tecnológica Federal do Paraná - Dois Vizinhos, PR \\ ${ }^{2}$ Universidade Federal de Santa Maria - Santa Maria, RS
}

\begin{abstract}
RESUMO
Avaliou-se a concentração de metabólitos sanguíneos de vacas de corte mantidas em pastagem natural recebendo suplementação com sais de cálcio de ácidos graxos (SCAG) durante 45 dias antes do parto (PRE), suplementação com SCAG durante 45 dias antes do parto e 63 dias pós-parto (PREPOS), suplementação com SCAG durante 63 dias pós-parto (POS) e de vacas não suplementadas (PN). As coletas de sangue foram realizadas em média 64 dias antes do parto, e aos 21, 42 e 63 dias pós-parto. Não ocorreu interação significativa entre tratamentos e períodos. As concentrações plasmáticas de $\beta$ hidroxibutirato $(\beta \mathrm{HB})$, triglicerídeos (TRIG), colesterol, glicose e ureia não foram afetadas significativamente pela suplementação de gordura protegida. A análise de regressão mostrou queda linear significativa da concentração de TRIG no sangue com o aumento da produção de leite (PL) para as vacas do tratamento PN (TRIG $\left.=23,10-2,18 * \mathrm{PL}, \mathrm{R}^{2}=0,31\right)$ e efeito quadrático para as vacas do PRE $(\mathrm{TRIG}=$ $\left.6,54-1,75 * \mathrm{PL}+0,30 * \mathrm{PL}^{2}, \mathrm{R}^{2}=0,62\right)$. Nos animais dos tratamentos POS e PREPOS, não houve efeito da produção de leite sobre a concentração de TRIG, indicando que a suplementação durante o período de produção de leite auxilia na manutenção de um balanço energético positivo. As concentrações de colesterol plasmático aumentaram, e as de triglicerídeos e ureia diminuíram linearmente até o final do experimento.
\end{abstract}

Palavras-chave: bovino, $\beta$-hidroxibutirato, colesterol, glicose, triglicerídeos, ureia

\begin{abstract}
The study evaluated the blood metabolites of beef cows maintained on native pasture supplemented with calcium salts of fatty acids (CSFA) during 45 days pre-partum (PRE); supplemented with CSFA during 45 days pre-partum and 63 days post-partum (PREPOS); supplemented with CSFA during 63 days postpartum (POS) and cows not supplemented (PN): without supplementation. Blood samples were taken 64 days pre-partum, and at 21, 42 and 63 days post-partum. No significant interaction was observed between treatment and period. Plasma concentrations of $\beta$-hydroxybutyrate ( $\beta H B)$, triglycerides, cholesterol, glucose and urea were not affected by protected fat supplementation. The regression analysis showed significant linear decline of blood triglycerides (TRIG) concentration with the increase of milk production $(P L)$ for $P N$ cows $\left(T R I G=23.10-2.18 * P L, R^{2}=0.31\right)$ and quadratic effect for PRE cows $\left(T R I G=6.54-1.75 * P L+0.30 * P L^{2}, R^{2}=0.62\right)$. For cows submitted to the POS and PREPOS treatments there was no effect of milk production on blood triglycerides concentration, indicating that CSFA during milk production aids the maintenance of a positive energetic balance. The concentration of plasma cholesterol increased while the concentrations of triglycerides and urea decreased linearly until the end of the experiment.
\end{abstract}

Keywords: cattle, $\beta$-hydroxybutyrate, cholesterol, glucose, triglycerides, urea

Recebido em 24 de março de 2011

Aceito em 28 de agosto de 2012

E-mail: mg_flor@yahoo.com.br 


\section{INTRODUÇÃO}

O rebanho de cria no Brasil é criado em condições extensivas, apresentando baixos índices reprodutivos, os quais estão relacionados a vários fatores, como nutrição, manejo, sanidade, genética, entre outros. O conhecimento das relações entre os níveis plasmáticos dos metabólitos sanguíneos, escore corporal e desempenho reprodutivo em vacas poderiam auxiliar o entendimento da sua fisiologia, fornecendo informações sobre a eficiência reprodutiva. Os metabólitos sanguíneos têm sido utilizados para indicar transtornos metabólicos que ocorrem durante o pós-parto em vacas, e também podem servir como indicadores do estado nutricional, quando dietas ricas em gordura são utilizadas. A suplementação pré e/ou pós-parto com lipídios pode contribuir para prevenir o balanço energético negativo, pelo aumento da concentração de glicose no plasma e diminuição das concentrações de $\beta$ hidroxibutirato $(\beta \mathrm{HB})$.

A glicose tem papel importante na produção de leite por suprir carbono, hidrogênio e oxigênio para a síntese de lactose, que é o maior regulador osmótico que controla o volume de leite produzido (Mühlbach et al., 2000). Além disso, a glicose é o primeiro nutriente metabólico utilizado pelo sistema nervoso central, e uma inadequada disponibilidade nas suas concentrações plasmáticas reduz a liberação de GnRH pelo hipotálamo, afetando os níveis de LH, uma vez que a secreção pulsátil de LH é associada com a secreção de GnRH (Gazal et al., 1998).

A suplementação com gordura aumenta as concentrações plasmáticas de colesterol (Williams, 1989; Hightshoe et al., 1991), beneficiando o desempenho reprodutivo, visto que o colesterol é o precursor de hormônios esteroides importantes, como a progesterona. A maioria do colesterol plasmático é transportada por lipoproteínas de alta (HDL) e baixa (LDL) densidade, que são apreendidas pelo tecido ovariano para a esterodeiogênese (Grummer e Carrol, 1991). De 25 a $55 \%$ dos embriões são perdidos durante o início da gestação (Staples et al. 1998), e o principal motivo é a inadequada síntese de progesterona pelas células do corpo lúteo.
Níveis plasmáticos de ureia são um indicador do metabolismo proteico em ruminantes. Sua concentração está diretamente relacionada aos níveis proteicos da dieta e da relação energia/proteína desta (Wittwer et al., 2000).

Portanto, o objetivo deste estudo é avaliar os efeitos da suplementação com sais de cálcio de ácidos graxos durante o período pré e/ou pósparto nos níveis plasmáticos dos metabólitos sanguíneos de vacas mantidas em pastagem natural.

\section{MATERIAL E MÉTODOS}

Foi estudado o efeito da suplementação de sais de cálcio de ácidos graxos (Megalac ${ }^{\circledR}-\mathrm{E}$, Química Geral do Nordeste) nos metabólitos sanguíneos de vacas de corte mantidas em pastagem natural, distribuídas nos seguintes tratamentos alimentares: PRE: suplementação com sais de cálcio de ácidos graxos (SCAG) 45 dias antes do parto; PREPOS: suplementação com SCAG 45 dias antes do parto e 63 dias pósparto; POS: suplementação com SCAG 63 dias pós-parto; PN: sem suplementação.

De um total de 86 vacas utilizadas no experimento, 24 foram selecionadas para a coleta de sangue, todas oriundas do mesmo rebanho experimental, cruzas Charolês-Nelore. O peso médio das vacas foi de 414, 438, 381, e $419 \mathrm{~kg}$ para o PRE, PREPOS, POS e PN, respectivamente. As vacas foram distribuídas nos tratamentos de acordo com grupo genético e idade, buscando manter a homogeneidade.

O suplemento utilizado foi sais de cálcio de ácidos graxos (Megalac ${ }^{\circledR}-\mathrm{E}$, Química Geral do Nordeste), fornecido juntamente com $80 \mathrm{~g}$ de sal mineral com $8 \%$ de fósforo, na quantidade diária de $60 \mathrm{~g} / \mathrm{vaca}$ durante o período pré-parto e $100 \mathrm{~g} /$ vaca durante o período pós-parto. O produto recomenda utilizar 50-120g/cabeça/dia três semanas antes do parto, e após o parto devese aumentar a dose para $150 \mathrm{~g} / \mathrm{cabeça/dia} \mathrm{até}$ atingir 250-900g/cabeça/dia. Na Tab. 1 encontrase a composição em ácidos graxos da gordura protegida comercial. No entanto, essa recomendação é para vacas de leite de alta produção. Como as vacas utilizadas no presente estudo são vacas de corte de baixa produção leiteira, foi realizado um ajuste para as vacas do presente experimento, utilizando-se a menor recomendação sugerida pela empresa. 
Tabela 1. Composição dos ácidos graxos ${ }^{1}$ da gordura protegida comercial oferecida durante o estudo

\begin{tabular}{lc}
\hline Ácidos graxos & $\%$ \\
\hline $\mathrm{C}_{12: 0}$ & 0,89 \\
$\mathrm{C}_{16: 0}$ & 13,85 \\
$\mathrm{C}_{18: 0}$ & 4,01 \\
$\mathrm{C}_{18: 1} \mathrm{n} 9 \mathrm{t}$ & 3,18 \\
$\mathrm{C}_{18: 1} \mathrm{n} 9 \mathrm{c}$ & 17,92 \\
$\mathrm{C}_{18: 2} \mathrm{n} 6 \mathrm{c}$ & 49,09 \\
$\mathrm{C}_{20: 0}$ & 0,84 \\
$\mathrm{C}_{18: 3} \mathrm{n}$ & 4,39 \\
$\mathrm{C}_{21: 0}$ & 1,80 \\
$\mathrm{C}_{22: 0}$ & 0,68 \\
Total de picos não identificados & 2,75 \\
\hline
\end{tabular}

${ }^{\mathrm{T}}$ Megalac E (Química Geral do Nordeste, Rio de Janeiro, Brasil)

A área experimental utilizada correspondeu a 88 hectares, divididos em três potreiros de 29,3 ha. A cada 14 dias, foi realizado o rodízio dos animais para minimizar o efeito do potreiro. A lotação média foi de $0,6 \mathrm{UA} / \mathrm{ha}$.

As coletas de sangue foram realizadas em média 64 dias antes do parto, e aos 21, 42 e 63 dias pósparto. As amostras de sangue das vacas foram coletadas da veia jugular em tubos Vacutainer ${ }^{\circledR}$ heparinizados de $10 \mathrm{~mL}$, devidamente identificados e mantidos refrigerados até o momento da centrifugação. Posteriormente, as amostras foram centrifugadas 5.000rpm por cinco minutos para a obtenção do plasma sanguíneo. O plasma foi armazenado em ependorfes de $2 \mathrm{~mL}$ e conservados a $-20^{\circ} \mathrm{C}$. As concentrações plasmáticas de glicose, triglicerídeos, colesterol, $\beta$-hidroxibutirato $(\beta \mathrm{HB})$ e ureia foram determinadas pelo método enzímico colorimétrico, utilizando kits comerciais (Glicose enzimática líquida, Doles®; Triglicérides enzimático líquido, Doles®; Colesterol 250, Doles®; Ureia 500, Doles $₫$; D3-Hydroxybutyrate, no FA 1007, Randox $\left.{ }^{\circledR}\right)$.

O delineamento experimental utilizado foi $\mathrm{o}$ inteiramente ao acaso, em esquema fatorial $4 \times 3$, quatro manejos alimentares e três períodos, com número variável de repetições. Os dados coletados foram submetidos à análise de variância, incluindo no modelo os efeitos da suplementação e período e as interações entre esses fatores. A ordem de parição e a idade da vaca foram incluídas no modelo como covariáveis. As médias quando significativamente diferentes foram comparadas pelo teste " $\mathrm{t}$ " em nível de $5 \%$ de significância. Adicionalmente, foram realizadas análise de regressão quando a análise de variância demonstrou efeito do período. Foi excluída da análise a coleta de sangue aos 64 dias antes do parto para a comparação entre tratamentos, uma vez que ainda não havia efeito de nenhum dos tratamentos. Todas as análises foram realizadas pelo programa estatístico SAS (2001).

\section{RESULTADOS E DISCUSSÃO}

Não houve interação significativa entre tratamentos e os períodos de avaliação nas variáveis estudadas. $\mathrm{Na}$ Tab. 2 verifica-se que a suplementação com sais de cálcio de ácidos graxos não alterou o escore de condição corporal (EC) e o peso vivo médio das vacas $(\mathrm{P}>0,05)$.

Tabela 2. Médias do escore corporal e peso vivo $(\mathrm{kg})$ de vacas de corte de acordo com o manejo alimentar

\begin{tabular}{lcccc}
\hline \multirow{2}{*}{ Variável } & \multicolumn{4}{c}{ Manejo alimentar } \\
\cline { 2 - 5 } & PN & PRE & POS & PREPOS \\
\hline Escore corporal ao parto & $2,63 \pm 0,09$ & $2,46 \pm 0,07$ & $2,51 \pm 0,05$ & $2,63 \pm 0,05$ \\
Peso vivo ao parto & $418,7 \pm 44,8$ & $413,8 \pm 30,8$ & $381,9 \pm 24,2$ & $438,1 \pm 24,7$ \\
Escore corporal médio & $2,64 \pm 0,15$ & $2,56 \pm 0,10$ & $2,65 \pm 0,08$ & $2,64 \pm 0,08$ \\
Peso vivo médio & $424,2 \pm 39,5$ & $421,8 \pm 27,1$ & $380,3 \pm 21,4$ & $438,5 \pm 21,8$ \\
\hline
\end{tabular}

PN: sem suplementação; PRE: suplementação com sais de cálcio de ácidos graxos (SCAG) 45 dias antes do parto; POS: suplementação SCAG 63 dias pós-parto; PREPOS: suplementação com sais de cálcio de ácidos graxos durante 45 dias antes do parto e 63 dias pós-parto. P>0,05. 
As concentrações plasmáticas de $\beta$ hidroxibutirato $(\beta \mathrm{HB})$ não diferiram entre os tratamentos $(\mathrm{P}>0,05)$, com média de $0,55 \mathrm{mmol} / \mathrm{L}$ (Tab. 3). O $\beta \mathrm{HB}$ é um produto fisiológico do metabolismo dos glicídios e lipídios de ruminantes, seus precursores são as gorduras e ácidos graxos da dieta, assim como os depósitos de gordura do animal. O ácido butírico da dieta é transformado no epitélio ruminal, via acetoacetato, em $\beta \mathrm{HB}$, sendo este o principal corpo cetônico do sangue de ruminantes (Wittwer, 2000).

Dessa forma, os resultados indicam que não houve mobilização dos ácidos graxos do tecido adiposo das vacas que receberam ou não suplementação, sendo que provavelmente o $\beta \mathrm{HB}$ foi proveniente da dieta. Esse metabólito mostra aumento relativamente pequeno em balanço energético moderado, mas apresenta um aumento considerável quando o balanço energético negativo torna-se severo (Russel e Wright, 1983). Grummer et al. (1995), ao avaliarem diferentes dietas fornecidas para vacas holandesas primíparas no pré e pós-parto, observaram interação significativa entre as dietas avaliadas no pré-parto e no pós-parto para a concentração de $\beta \mathrm{HB}$ no plasma. Isso indica que as vacas suplementadas com gordura no pós-parto apresentaram maior concentração de $\beta \mathrm{HB}$ no plasma quando estas foram alimentadas com uma dieta padrão no pré-parto, e menor concentração de $\beta \mathrm{HB}$ quando as vacas foram suplementadas com alta energia no pré-parto. Segundo Grummer e Carrol (1991), a suplementação com gordura mantém relativamente estáveis ou diminui as concentrações de $\beta \mathrm{HB}$, sugerindo um efeito anticetogênico de dietas ricas em gordura.

Tabela 3. Concentrações médias dos metabólitos sanguíneos de vacas de corte de acordo com o manejo alimentar

\begin{tabular}{ccccc}
\hline \multirow{2}{*}{ Variável } & \multicolumn{4}{c}{ Manejo Alimentar } \\
\cline { 2 - 5 } & $\mathrm{PN}$ & PRE & POS & PREPOS \\
\hline$\beta \mathrm{HB}, \mathrm{mmol} / \mathrm{L}$ & $0,45 \pm 0,11$ & $0,57 \pm 0,06$ & $0,59 \pm 0,06$ & $0,57 \pm 0,08$ \\
Triglicerídeos, mg/dL & $5,96 \pm 2,11$ & $6,85 \pm 1,52$ & $7,84 \pm 1,23$ & $9,05 \pm 1,21$ \\
Colesterol, mg/dL & $159,05 \pm 52,29$ & $193,53 \pm 42,63$ & $251,18 \pm 31,58$ & $242,74 \pm 28,84$ \\
Glicose, $\mathrm{mg} / \mathrm{dL}$ & $62,26 \pm 6,89$ & $60,26 \pm 4,79$ & $60,71 \pm 3,82$ & $60,84 \pm 4,39$ \\
Ureia, mg/dL & $32,01 \pm 5,49$ & $28,89 \pm 4,39$ & $34,25 \pm 3,18$ & $27,41 \pm 3,01$ \\
\hline
\end{tabular}

$\beta \mathrm{HB}=$ beta-hidroxiburitato;

PN: sem suplementação; PRE: suplementação com sais de cálcio de ácidos graxos (SCAG) 45 dias antes do parto; POS: suplementação SCAG 63 dias pós-parto; PREPOS: suplementação com sais de cálcio de ácidos graxos durante 45 dias antes do parto e 63 dias pós-parto.

$\mathrm{P}>0,05$.

As concentrações de triglicerídeos foram similares $(\mathrm{P}>0,05)$ entre as vacas que receberam a suplementação com gordura no PRE, POS, PREPOS, e as vacas não suplementadas (Tab. 3). Porém, a análise de regressão entre a produção de leite (PL) e as concentrações de triglicerídeos (TRIG) foi significativa para as vacas dos tratamentos PN e PRE (TRIG $=23,10-$ $2,18 * \mathrm{PL}, \mathrm{R}^{2}=0,31 ; \mathrm{TRIG}=6,54-1,75 * \mathrm{PL}+$ $0,30 * \mathrm{PL}^{2}, \mathrm{R}^{2}=0,62$, respectivamente). Quanto às vacas da $\mathrm{PN}$, para cada litro de leite produzido houve uma diminuição de $2,18 \mathrm{mg} / \mathrm{dL}$ na concentração de triglicerídeos no sangue, indicando que, para a síntese de leite, as vacas não suplementadas utilizaram os lipídios do sangue. Já para as vacas do PRE, houve efeito quadrático na análise de regressão. Isso indica que as vacas que não receberam suplementação de SCAG no pós-parto provavelmente utilizaram os lipídios do sangue em maior proporção para a síntese de leite na glândula mamária, enquanto que as vacas suplementadas no período pós-parto utilizaram os lipídios da dieta. Tanto o escore de condição corporal como o peso vivo das vacas não foram afetados pelos tratamentos (Tab. 1), indicando que não houve mobilização de tecidos para manter a lactação.

Nos animais que receberam suplementação no pós-parto (POS e PREPOS), não houve efeito da produção de leite sobre a concentração de triglicerídeos, indicando que a suplementação durante o período de produção de leite auxilia na manutenção de um balanço energético positivo. 
Avaliando a inclusão de sais de cálcio de ácidos graxos na dieta de vacas holandesas, Canale et al. (1990) observaram aumento na concentração de triglicerídeos no plasma sanguíneo (130,2 vs $114,5 \mu \mathrm{eq} / \mathrm{L}$ ) das vacas que receberam gordura suplementar. Já Bermudes et al. (2003) não verificaram diferença nas concentrações plasmáticas de triglicerídeos quando vacas leiteiras foram suplementadas ou não com 400 gramas de gordura protegida na ração, apresentando concentrações médias de 19,9 vs 20,1mg/\%, respectivamente. Williams (1989) verificou incremento de 1,4 vezes nas concentrações de triglicerídeos na quarta semana após o parto de vacas Brahman com cria ao pé comparada ao controle. Esse mesmo autor relata que os triglicerídeos não apresentam um papel importante na esteroidogênese ovariana, mas servem como indicador útil juntamente com o colesterol, no status geral do metabolismo lipídico. A quantidade de gordura fornecida no presente estudo, tanto no período pré como no pós-parto, ou em ambos, aparentemente não foi suficiente para aumentar as concentrações de triglicerídeos.

A suplementação com gordura no período pré e/ou pós-parto não afetaram $(\mathrm{P}>0,05)$ as concentrações plasmáticas de colesterol total (Tab. 3); porém, observa-se uma tendência no aumento das concentrações no sangue das vacas suplementadas. Provavelmente, esse aumento está relacionado ao consumo de gordura. Alguns autores verificaram aumento dos níveis de colesterol em vacas, à medida que o EC diminui (Ruegg, 1992; Ruas et al., 2000a), relacionado ao fato de mobilizarem reservas corporais para atenderem à demanda de nutrientes para a lactação e mantença. Como não houve alteração no EC das vacas, os níveis de colesterol foram semelhantes. O colesterol é um componente das membranas celulares e é o precursor para a síntese de hormônios esteroides, entre eles, a progesterona (Grummer e Carrol, 1991).

Grande parte do colesterol sanguíneo é transportada por lipoproteínas de alta (HDL) e baixa (LDL) densidade, que são utilizadas pelo tecido ovariano para a síntese desses hormônios (Grummer e Carrol, 1988). Ruas et al. (2000b) não observaram diferença significativa nas concentrações de colesterol entre vacas suplementadas ou não no pós-parto, com média de $182,34 \mathrm{mg} / \mathrm{dL}$; resultado similar foi encontrado por Godoy et al. (2004). Diferenças nas concentrações de colesterol plasmático foram verificadas por Hightshoe et al. (1991), suplementando vacas com SCAG pós-parto, em relação às vacas que não receberam gordura na dieta.

As concentrações de glicose foram semelhantes ( $\mathrm{P}>0,05)$ entre os tratamentos (Tab. 3). A glicose é um importante substrato metabólico, requerido para manter as funções adequadas para $\mathrm{o}$ processo reprodutivo. A glicose é o primeiro nutriente metabólico usado pelo sistema nervoso central (SNC), e inadequada disponibilidade de glicose reduz a liberação hipotalâmica de $\mathrm{GnRH}$ (Wetteman et al., 2003). Os ruminantes absorvem pouca glicose a partir do trato digestivo, devido à extensa degradação dos carboidratos do alimento em ácidos graxos voláteis no rúmen; sendo que os principais substratos para a síntese de glicose em ruminantes são o propionato e aminoácidos gliconeogênicos (Clark, 1975). Experimento usando glicose infundida (Garmmendia et al., 1986) em vacas de corte aumentou a concentração de insulina e diminuiu a lipólise em resposta à infusão de glicose. Outra função importante da glicose é na síntese de lactose do leite, suprindo carbono, hidrogênio e oxigênio para sua síntese. Em torno de 60 a $85 \%$ da glicose disponível no corpo é utilizada pela glândula mamária. E destes, 80 a $85 \%$ são usados para a síntese de lactose (Clark, 1975). Aparentemente a suplementação com gordura protegida responde de maneira variável nas concentrações plasmáticas de glicose. Jenkis e Jenny (1989) e Gagliostro et al. (1991) observaram aumento nas concentrações de glicose, já Bermudes et al. (2003) não verificaram o mesmo ao suplementar com lipídios protegidos.

A ureia é um produto da excreção do metabolismo do nitrogênio, e a sua determinação revela informações sobre a atividade proteica do animal. A suplementação com gordura para vacas de corte durante o pré-parto, pós-parto e no pré e pós-parto $(29,81 ; 35,16$ e $30,85 \mathrm{mg} / \mathrm{dL}$, respectivamente) apresentaram concentrações semelhantes de ureia às de vacas não suplementadas (34,71 mg/dL) (Tab. 3). Estimase que dietas com menos de $10 \%$ de proteína causam diminuição dos níveis proteicos no sangue (Kaneco et al., 1997). 
As concentrações médias de colesterol aumentaram linearmente até o final do experimento (Fig. 1). O aumento de colesterol pode estar associado à mobilização das reservas corporais para atender à exigência de nutrientes para a mantença e lactação. Porém, não houve alteração na condição corporal (EC) das vacas ao longo do período, com média de 2,57 pontos. É provável que esse aumento nos níveis de colesterol deva-se ao aumento da disponibilidade e da condição nutricional da pastagem.

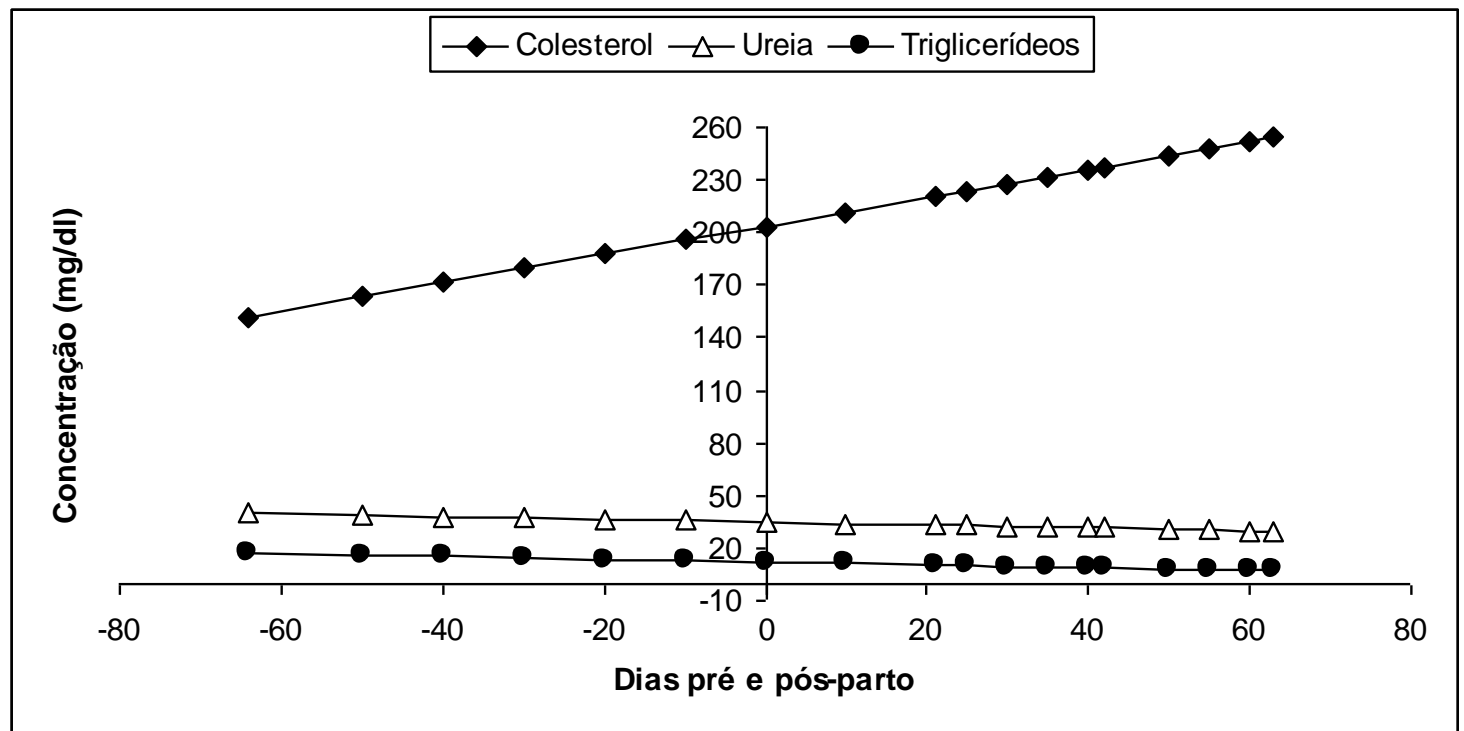

Colesterol = 205,77+0,829*Dias; $\mathrm{R}^{2}=0,27(\mathrm{P}<0,01)$; Triglicerídeos $=11,97-0,085^{*}$ Dias; $\mathrm{R}^{2}=0,50(\mathrm{P}<0,01)$; Ureia $=34,63-0,082 *$ Dias; $\mathrm{R}^{2}=0,11(\mathrm{P}=0,0070)$.

Figura 1. Concentrações de colesterol, triglicerídeos e ureia $(\mathrm{mg} / \mathrm{dL})$ no plasma sanguíneo de vacas de corte de acordo com o período.

Ruas et al. (2000a), ao avaliar o efeito da EC das vacas sobre a concentração de colesterol durante o período pós-parto, observaram que, nos primeiros dias após o parto (0-45 dias), as concentrações foram mais baixas que as dos demais períodos avaliados (46-90 dias e acima de 90 dias). Esses autores atribuíram o fato à menor mobilização de gordura no pós-parto imediato; porém, com o avançar dos dias pósparto, a EC diminui, indicando mobilização de tecidos, aumentando as concentrações de colesterol circulante.

Os valores médios de colesterol sanguíneo foi de $152,11 \mathrm{mg} / \mathrm{dL}$ aos 64 dias antes do parto, $203,63 \mathrm{mg} / \mathrm{dL}$ no parto e $254,34 \mathrm{mg} / \mathrm{dL}$ aos 63 dias pós-parto. $\mathrm{O}$ aumento na concentração de colesterol com o avanço dos dias pós-parto tem mostrado uma tendência geral na literatura. Godoy et al. (2004) encontraram valores médios para vacas suplementadas ou não, oscilando de 108,05 a $118,27 \mathrm{mg} / \mathrm{dL}$ ao parto e 174,22 a
182,71 aos 112 dias pós-parto, respectivamente, e Carrol et al. (1990), 134 a $199 \mathrm{mg} / \mathrm{dl}$ para os primeiros 100 dias de lactação. Além disso, animais alimentados com dietas ricas em gordura apresentam concentrações plasmáticas mais elevadas de colesterol. Gagliostro e Chilliard (1992) verificaram que o aumento das concentrações de lipídios totais no plasma pela suplementação com lipídios foi relatado de forma consistente na literatura como consequência do aumento de todas as frações lipoproteicas do plasma.

Os níveis plasmáticos de triglicerídeos diminuíram linearmente (TRIG $=11,97-$ $0,085 *$ Dias; $\left.R^{2}=0,50\right)$ com o avanço do período experimental (Fig. 1). Essa diminuição pode ter sido causada pela utilização direta dos triglicerídeos pela glândula mamária. Isso ocorre pelo melhor aproveitamento dos ácidos graxos de cadeia longa da dieta para a síntese do leite na glândula mamária, disponibilizando maior 
quantidade de glicose para as células produtoras de leite (Nörnberg et al., 2006). Palmquist e Jenkis (1980) observaram que a relativa contribuição exógena (quilomicrons a partir da gordura da dieta) e endógena (VLDL sintetizado no fígado) dos triglicerídeos para a síntese de gordura no leite varia com o estado fisiológico do animal. Portanto, no início da lactação, quantidades significativas de ácidos graxos do tecido adiposo são mobilizadas, sendo que a contribuição endógena é relativamente maior, fato explicado pela redução de triglicerídeos no sangue das vacas do presente estudo. Williams (1989) verificou aumento nos níveis de triglicerídeos do parto até oito semanas pós-parto em vacas Brahman, declinando após esse período. Hightshoe et al. (1991) verificaram similaridade nas concentrações plasmáticas de triglicerídeos durante o período experimental entre as vacas suplementadas com SCAG e não suplementadas $\quad(13,7 \quad$ e $12,7 \mathrm{mg} / \mathrm{dL}$, respectivamente).

Os valores médios da concentração de ureia no sangue apresentaram diminuição linear com o transcorrer do período experimental (Fig. 1). A concentração de ureia no plasma sanguíneo é diretamente proporcional à taxa de amônia no rúmen (Kennedy e Milligan, 1980). Isso sugere que, com o transcorrer do período experimental, houve variações nas produções de amônia no rúmen, ocorridas talvez pela diminuição de proteína bruta da dieta, ou pela melhor sincronização entre energia:proteína da pastagem. Além disso, para Ferguson e Chalupa (1989), outros fatores, além da deficiência de proteína, podem alterar a concentração de ureia em vacas, como a degradabilidade da proteína, o consumo energético, a taxa de degradação, o tempo da coleta após a alimentação e os métodos de suplementação.

As médias para os níveis de ureia no sangue das vacas foram de 39,88, 34,76 e 29,72mg/dL para 64 dias pré-parto, ao parto e 63 dias pós-parto, respectivamente. Apenas o valor médio de ureia aos 63 dias pós-parto não se encontra dentro dos valores preconizados por Contreras (2000) para bovinos $(36-126 \mathrm{mg} / \mathrm{dL})$. Os resultados do presente estudo discordam dos encontrados por Peixoto et al. (2006), os quais suplementaram vacas de corte em pastagem natural com farelo de trigo e farelo de trigo mais ureia na proporção de $0,7 \% \mathrm{PV}$, e verificaram que as concentrações de nitrogênio ureico sofreram variações, com os valores decrescendo dos 21 aos 42 dias pós-parto e voltando a subir aos 63 dias pós-parto.

Ruas et al. (2000a) verificaram reduções nas concentrações de ureia plasmática em vacas Nelore, à medida que aumentaram os dias pósparto, com média de $21,2 \mathrm{mg} / \mathrm{dL}$ aos 62 dias e $12,11 \mathrm{mg} / \mathrm{dL}$ aos 82 dias após o parto, atribuindo à mobilização de tecidos de reserva.

\section{CONCLUSÕES}

A suplementação com sais de cálcio de ácidos graxos durante o período pré e/ou pós-parto não afetou os metabólitos sanguíneos de vacas de corte. Vacas mantidas em pastagem natural e as suplementadas durante o pré-parto utilizaram os triglicerídeos sanguíneos para a síntese de leite na glândula mamária. Com o transcorrer dos dias do período experimental, os níveis de colesterol aumentaram e os níveis plasmáticos de triglicerídeos e ureia diminuíram.

\section{REFERÊNCIAS}

BERMUDES, R.F.; LÓPEZ, J.; GALLARDO, M. et al. Gordura protegida na dieta de vacas de alta produção a campo, em alfafa verde ou présecada, na fase inicial de lactação. Parâmetros plasmáticos. Rev. Bras. Zootec., v.32, p.405-410, 2003.

CANALE, C.J.; MULLER, L.D.; McCAHON, H.A. et al. Dietary fat and ruminally protected amino acids for high producing dairy cows. $J$. Dairy Sci., v.73, p.135-141, 1990.

CARROL, D.J.; JERRED, M.J.; GRUMMER, R.R. et al. Effects of fat supplementation and immature alfafa to concentrate ratio on plasma progesterone, energy balance, and reproductive traits of dairy catlle. J. Dairy Sci., v.73, p.28552863, 1990.

CLARK, J.H. Lactational responses to postruminal administration of proteins and amino acids. J. Dairy Sci., v.58, p.1178-1197, 1975.

CONTRERAS, P.A. Indicadores do metabolismo proteico utilizados nos perfis metabólicos de rebanhos. In: GONZÁLEZ, F.H.D., BARCELlOS, J., PATIÑO, H.O. et al. (Eds). Perfil metabólico em ruminantes: Seu uso em nutrição e doenças nutricionais. Porto Alegre:UFRGS, 2000. p.23-30. 
FERGUSON, J.D.; CHALUPA, W. Symposium: interactions of nutrition and reproduction. $J$. Dairy Scie., v.72, p.746-766, 1989.

GAGLIOSTRO, G.A.; CHILLIARD, Y. Utilizácion de lipídios protegidos em la nutricion de la vaca lechera. II. Efectos sobre la concentracion plasmática de metabolitos e hormonas, mobilizacion de lipídios corporales y actividad metabólica del tejido adiposo. Rev. Argentina de Produción Anim, v.12, p.17-32, 1992.

GAGLIOSTRO, G.A.; CHILLIARD, Y.; DAVICCO, M.J. Duodenal rapeseed oil infusion in early and midlactation cows. 3. Plasma hormones and mammary apparent uptake metabolites. J. Dairy Sci., v.74, p.1893-1903, 1991.

GAZAL, O.S.; LESHIN, L.S; STANKO, R.L. et al. Gonadotropin-releasing hormone secretion into third-ventricle cerebrospinal fluid in cattle: Correspondence with the tonic and surge release of luteinizing hormone and its tonic inhibition by suckling and Neuropeptide Y. Biol. Reprod., v.59, p.676-683, 1998.

GODOY, M.M.; ALVES, J.B.; MONTEIRO, A.L.G. et al. Parâmetros reprodutivos e metabólicos de vacas da raça Guzerá suplementadas no pré e pós-parto. Rev. Bras. Zootec., v.33, p.103-111, 2004.

GRUMMER, R.R.; CARROL, D.J. Effects of dietary fat on metabolic disorders and reproductive performance of dairy cattle. $J$. Dairy Sci., v.69, p.3838-3852, 1991.

GRUMMER, R.R; CARROL, D.J. A review of lipoprotein cholesterol metabolism:importance to ovarian function. J. Dairy Sci., v.66, p.31603173, 1988.

GRUMMER, R.R.; HOFFMAN, P.C.; LUCK, M.L. Effects of prepartum and postpartum dietary energy on growth and lactation of primiparous cows. J. Dairy Sci., v.78, p.172$180,1995$.
HIGHTSHOE, R.B.; COCHRAN, R.C.; CORAH, L.R. et al. Effects of calcium soaps of fatty acids on postpartum reproductive function in beef cows. J. Anim. Sci., v.69, p.4097-4103, 1991.

JENKIS, T.C.; JENNY, B.F. Effects of hydrogenated fat intake, nutrient digestion, and lactation performance of dairy cows. J. Dairy Sci., v.72, p.2316-2324, 1989.

KANECO, J.J.; HARVEY, J.W.; BRUSS, M.L. Clinical Biochemistry of domestic animals, 5nd ed. California: Academic, 1997. 932p.

KENNEDY, P.M.; MILLIGAN, L.P. The degradation and utilization of endogenous urea in the gastrointestinal tract of ruminants: a review. Canadian J. Anim. Sci., v.60, p.205-221, 1980.

MÜHLBACH, P.R.F.; OSPINA, H.; PRATES, E.R.; BARCELLOS, J.O.J. Aspectos nutricionais que interferem na qualidade do leite. In: ENCONTRO ANUAL DA UFRGS SOBRE NUTRIÇÃO DE RUMINANTES, 2., 2000. Porto Alegre. Anais... Novos desafios para a produção leiteira do Rio Grande do Sul. Porto Alegre: Departamento de Zootecnia da UFRGS, 2000. 73-102.

NÖRNBERG, J.L.; LÓPEZ, J.; STUMPF JÚNIOR, W. et al. Desempenho de vacas Jersey suplementadas com diferentes fontes lipídicas na fase inicial de lactação. Rev. Bras. Zootec., v.35, p.1431-1438, 2006.

PALMQUIST, D.L.; JENKIS, T.C. Fat in lactation rations: review. J. Dairy Sci., v.63, p.114, 1980.

PEIXOTO, L.A.O; BRONDANI, I.L.; NÖRNBERG, J.L. et al. Perfil metabólico proteico e taxas de concepção de vacas de corte mantidas em pastagem natural ou suplementadas com farelo de trigo com e sem ureia. Cienc. Rural, v.36, p.1873-1877, 2006.

RUAS, J.R.M.; TORRES, C.A.A.; BORGES, L.E. et al. Efeito da suplementação proteica a pasto sobre a eficiência reprodutiva $\mathrm{e}$ concentrações sanguíneas de colesterol, glicose e ureia, em vacas Nelore. Rev. Bras. Zootec., v.29, Supl.1, p.2043-2050, 2000a. 
RUAS, J.R.M.; TORRES, C.A.A.; BORGES, L.E. et al. Concentrações plasmáticas de colesterol, glicose e ureia em vacas Zebuínas, em relação à condição corporal ao status reprodutivo. Rev. Bras. Zootec., v.29, Supl.1, p.2036-2042, 2000b.

RUEGG, P.L.; GOODGER, W.J.; HOLMBERG, C.A. et al. Relation among body condition score, milk production and serum urea nitrogen and cholesterol concentrations in highproducing Holstein dairy cows in early lactation. Anim. J. Vet. Res., v.53, p.5-9, 1992a.

RUSSEL, A.J.F.; WRIGHT, I.A. The use of blood metabolites in the determination of energy status in beef cows. Anim. Product., v.37, p.335343, 1983.

SAS. Statistical Analysis Systems User's Guide. Version 2001, SAS Institute, Cary, NC.
STAPLES, R.C.; BURKE, J.M.; THATCHER, W.W. Influence of supplemental fats on reproductive tissues and performance of lactating cows. J. Dairy Sci., v.81, p.856-871, 1998.

WETTEMANN, R.P.; LENTS, C.A.; CICCIOLI, N.H. et al. Nutritional and suckling mediated anovulation in beef cows. J. Anim. Sci., v.81, p.48-59, 2003

WILLIAMS, G.L. Modulations of luteal activity in postpartum beef cows through changes in dietary lipid. J. Anim. Sci., v.67, p.785-793, 1989.

WITTWER, F. Diagnóstico dos desequilíbrios metabólicos de energia em rebanhos bovinos. In: GONZÁLEZ, F.H.D.; BARCELLOS, J.; PATIÑO, H.O. et al. (Eds.) Perfil metabólico em ruminantes: seu uso em nutrição e doenças nutricionais. Porto Alegre: UFRGS, 2000. p.922. 